\title{
Right ventricular diastolic dysfunction in patients with anticardiolipin antibodies and antiphospholipid syndrome
}

M G Tektonidou, J P A Ioannidis, I Moyssakis, K A Boki, V Vassiliou, P G Vlachoyiannopoulos, M K Kyriakidis, H M Moutsopoulos

\begin{abstract}
Objective-To evaluate the prevalence of diastolic dysfunction in patients with anticardiolipin antibodies (aCL) and to examine whether the antiphospholipid syndrome (APS) is associated with diastolic dysfunction independently of valvular abnormalities and systolic dysfunction. Methods-Pulsed, continuous, colour Doppler echocardiography was performed in 179 subjects, of whom 15 were excluded from the analysis because of systolic dysfunction or severe valvular disease. The remaining 164 subjects included 29 patients with primary APS, 26 patients with secondary APS (APS in the presence of systemic lupus erythematosus (SLE)), and 30 patients with SLE and aCL but without APS; 43 patients with SLE without $\mathrm{aCL}$ and 36 normal volunteers served as control groups.
\end{abstract}

Results-The groups compared differed significantly in all measures of right ventricular function. There was a gradation of increasing diastolic function impairment as manifested by prolonged deceleration time (DT) and isovolumic relaxation time (IVRT) across the groups of patients with SLE without aCL, SLE with aCL, secondary APS, and primary APS. Differences in left ventricular diastolic function measures were less prominent. In regression analysis, DT increased by $19.6 \mathrm{~ms}(p=0.002)$ in the presence of primary APS and by $20.1 \mathrm{~ms}$ $(p=0.038)$ in the presence of pulmonary hypertension. The titre of IgG aCL was the strongest predictor of a prolonged IVRT. Conclusion-Diastolic dysfunction, in particular of the right ventricle-that is, independent of valvular disease and systolic dysfunction, is a prominent feature of APS and may be related to the pathogenesis of the syndrome.

(Ann Rheum Dis 2001;60:43-48)

The association between anticardiolipin antibodies (aCL) ${ }^{1}$ and cardiac disease in the presence of systemic lupus erythematosus (SLE) or the antiphospholipid syndrome (APS) ${ }^{2}$ has been reported in various clinical studies. The earliest reports were of valvular disease, including verrucous valvular thickening, global valvular thickening, and mitral or aortic regurgitation..$^{3-6}$ Coronary artery occlusion and intracardiac thrombosis were later described..$^{7-9}$
There is also some evidence that aCL may also be accompanied by a decrease in the systolic and diastolic function of the left ventricle. ${ }^{10}{ }^{11}$ Probably, some of this effect may be independent of valvular disease, but there is limited evidence so far. Furthermore, there is no knowledge of whether diastolic function of the right ventricle may also be affected.

This study aimed at evaluating the prevalence of echocardiographically documented diastolic dysfunction of the right and left ventricles in patients with aCL with and without SLE and with and without APS in the absence of systolic dysfunction and significant valvular disease.

\section{Methods}

SUBJECTS

One hundred and seventy nine subjects were evaluated by echocardiography. Patients with aCL were divided into three different groups: (a) 30 patients with primary APS defined by the presence of aCL (or lupus anticoagulant) and at least two of the following: recurrent abortions, haemolytic anaemia, venous or arterial thrombosis, thrombocytopenia, livedo reticularis, or leg ulcers ${ }^{12}$; (b) 29 patients with APS secondary to SLE defined by the presence of at least four of the revised criteria of the American Rheumatism Association for the classification of $\mathrm{SLE}^{13}$; and (c) 34 patients with aCL in the setting of SLE, but without APS. We also considered two control groups of patients without aCL. The first group $(n=50)$ was randomly selected from among patients with SLE without aCL who are followed up in our clinic. The second control group comprised 36 subjects, matched for age and sex, without any immunological and cardiac disease, who had normal blood pressure, electrocardiogram, and Doppler echocardiography.

Exclusion criteria for all groups were documented myocardial infarction, previous history of rheumatic fever, alcoholism, and diabetes mellitus. Patients with severe valvular disease or decreased right ventricular (RV) and left ventricular (LV) systolic function on echocardiography were also excluded from the analysis. Patients with hypertension were included in the analysis of the data, but hypertension was considered as an adjusting factor in the multivariate regressions. The results were largely similar when the analysis was limited to patients without systemic hypertension (not reported). 
Table 1 Characteristics of compared groups

\begin{tabular}{|c|c|c|c|c|c|c|c|c|c|}
\hline \multirow[b]{3}{*}{ Group } & \multirow[b]{3}{*}{$\operatorname{Sex}(F)$} & \multirow{3}{*}{$\begin{array}{l}\text { Age mean } \\
(S D) \text { (years) }\end{array}$} & \multirow{3}{*}{$\begin{array}{l}\text { Disease duration } \\
\text { mean (SD) } \\
\text { (months) }\end{array}$} & \multicolumn{3}{|c|}{ Autoantibodies } & \multirow[b]{3}{*}{$M V R^{\star}$} & \multirow{3}{*}{$\begin{array}{l}\text { Any valvular } \\
\text { disease }\end{array}$} & \multirow{3}{*}{$\begin{array}{l}\text { Pulmonary } \\
\text { hypertensiont }\end{array}$} \\
\hline & & & & \multicolumn{2}{|c|}{ Cardiolipin } & \multirow[b]{2}{*}{$\beta_{2} G P I^{\star}$} & & & \\
\hline & & & & $I g G$ & $I g M$ & & & & \\
\hline pAPS $\star$ & $22 / 29$ & $41.4(10.3)$ & $90(86)$ & $26 / 29$ & $15 / 29$ & $17 / 29$ & $14 / 29$ & $14 / 29$ & $4 / 29$ \\
\hline SLE/APS* & $21 / 26$ & $36.9(13.0)$ & $115(89)$ & $20 / 26$ & $10 / 26$ & $21 / 26$ & $9 / 26$ & $10 / 26$ & $5 / 26$ \\
\hline $\mathrm{SLE} / \mathrm{aCL}{ }^{\star}$ & $27 / 30$ & $39.3(12.6)$ & $85(62)$ & $28 / 30$ & $9 / 30$ & $5 / 30$ & $13 / 30$ & $13 / 30$ & $1 / 30$ \\
\hline SLE/no aCL* & $41 / 43$ & $38.0(14.6)$ & $112(109)$ & $0 / 43$ & $0 / 43$ & $0 / 43$ & $8 / 43$ & $11 / 43$ & $4 / 43$ \\
\hline Normal & $31 / 36$ & $38.3(12.4)$ & ND & $0 / 36$ & $0 / 36$ & $0 / 36$ & $0 / 36$ & $0 / 36$ & $0 / 36$ \\
\hline
\end{tabular}

$\star \beta_{2}$ GPI $=\beta_{2}$ glycoprotein I; MVR = mitral valve regurgitation; SLE = systemic lupus erythematosus; APS = antiphospholipid syndrome; pAPS = primary APS; SLE/APS: APS secondary to SLE; SLE/aCL = SLE with aCL (without APS); SLE/no aCL = SLE without aCL or APS; ND = no disease.

†Pulmonary artery pressure above $30 \mathrm{~mm} \mathrm{Hg}$

ECHOCARDIOGRAPHIC EVALUATION

Comprehensive echocardiographic examination with pulsed, continuous, colour Doppler was performed with a Hewlet Packard sonos 1000 ultrasound system, using a $2.5 \mathrm{MHz}$ transducer. Four-chamber views were obtained. Those performing and evaluating the echocardiograms were unaware of the clinical diagnosis of the subjects. Left ventricle end systolic and end diastolic diameters, as well as interventricular septum and posterior wall thickness at end diastole, were measured for calculation of the fractional shortening (FS) and LV mass with the modified formula of Devereux et al. ${ }^{1415}$ An FS less than 29\% was considered as LV systolic dysfunction. RV systolic function was evaluated from the RV $\mathrm{dP} / \mathrm{dt}$ taken from the tricuspid regurgitation velocity signal and from the tricuspid annular plane systolic excursion (TAPSE), and was considered as impaired when $\mathrm{RV} \mathrm{dP} / \mathrm{dt}$ was $<220 \mathrm{~mm} \mathrm{Hg} / \mathrm{s}$ and TAPSE was $<12.5 \mathrm{~mm}$. $^{16-18}$ The $\mathrm{RV} \mathrm{dP/dt} \mathrm{could} \mathrm{be} \mathrm{measured} \mathrm{accurately} \mathrm{in}$ $24 \%$ of our patients. In the remaining patients the RV systolic function was assessed only by TAPSE. The two methods have high concordance $(r=0.45),{ }^{16}$ and we found no major differences in any of the patients where both methods could be applied.

When pulsed Doppler from the mitral and tricuspid inflow velocity curve was used the following variables were calculated: peak early velocity (E wave), peak velocity at the time of atrial contraction (A wave), E/A ratio, deceleration time (DT) of the peak early velocity and

Table 2 Right ventricular function parameters

\begin{tabular}{|c|c|c|c|c|c|}
\hline \multirow[b]{2}{*}{ Patient groups ${ }^{\star}$} & \multicolumn{5}{|c|}{ Echocardiographic parameters: mean (SD) } \\
\hline & $I V R T^{\star}$ (ms) & $E / A^{\star}$ & $E(\mathrm{~cm})$ & $A(\mathrm{~cm})$ & $D T$ (ms) \\
\hline pAPS & $58.8(9.4)$ & $0.93(0.15)$ & $48.9(8.0)$ & $53.5(10.4)$ & $167.9(27.2)$ \\
\hline SLE/APS & $54.2(11.4)$ & $0.93(0.13)$ & $50.5(9.4)$ & $55.2(10.4)$ & $152.7(30.0)$ \\
\hline $\mathrm{SLE} / \mathrm{aCL}$ & $55.7(9.8)$ & $1.02(0.15)$ & $55.0(9.5)$ & $55.1(15.8)$ & $147.7(27.9)$ \\
\hline $\mathrm{SLE} /$ no aCL & $51.9(9.6)$ & $1.03(0.18)$ & $55.5(8.4)$ & $55.2(10.7)$ & $137.6(22.5)$ \\
\hline Normal & $47.9(4.8)$ & $1.20(0.27)$ & $50.6(7.9)$ & $43.1(7.1)$ & $123.1(9.2)$ \\
\hline Comparisons & $p$ Values & & & & \\
\hline All five groups & 0.0001 & 0.0001 & 0.0045 & $<0.0001$ & $<0.0001$ \\
\hline First four groups & 0.041 & 0.012 & 0.0054 & 0.94 & 0.0001 \\
\hline First three groups & 0.23 & 0.026 & 0.030 & 0.85 & 0.021 \\
\hline First two groups & 0.10 & 0.93 & 0.51 & 0.56 & 0.053 \\
\hline \multicolumn{6}{|l|}{ Among patients: } \\
\hline \multicolumn{6}{|c|}{ Among patients with aCL: } \\
\hline APS $v$ no APS & 0.71 & 0.007 & 0.010 & 0.77 & 0.049 \\
\hline
\end{tabular}

${ }^{\star} \mathrm{aCL}=$ anticardiolipin antibodies; SLE $=$ systemic lupus erythematosus; APS = antiphospholipid syndrome; $\mathrm{pAPS}=$ primary APS; SLE/APS = APS secondary to SLE; SLE/aCL = SLE with aCL (without APS); SLE/no aCL = SLE without aCL or APS; IVRT = isovolumic relaxation time; E/A = ratio of $\mathrm{E}$ wave to $\mathrm{A}$ wave; $\mathrm{DT}$ = deceleration time; for definitions of groups of subjects see "Methods". the LV isovolumic relaxation time (IVRT), with simultaneous recording of the LV inflow and outflow velocities. The RV IVRT was defined as the time interval between the closure of the pulmonary valve and opening of the tricuspid valve. This was estimated by subtracting the time interval between the peak of the $\mathrm{R}$ wave on the electrocardiogram and the end of the pulmonary systolic flow profile from the interval between the peak of the $R$ wave and the onset of the tricuspid valve opening. At least three beats from the end inspiration and three beats from the end expiration were recorded, and their values were averaged. Pulmonary artery systolic pressure was estimated by continuous wave Doppler echocardiograms recorded in the apical four-chamber view as the peak systolic pressure gradient across the tricuspid valve (peak regurgitation velocity) plus the estimated right atrial pressure. ${ }^{19-22}$

Intraobserver and interobserver variability of the RV E wave, A wave, DT, and IVRT measurements were determined on 25 patients and were found to be low $(7.2 \%$ and $6.5 \%$ for the $\mathrm{E}$ wave, $6.9 \%$ and $6.3 \%$ for the A wave, $5.9 \%$ and $5.6 \%$ for DT, and $5.4 \%$ and $5.1 \%$ for IVRT).

Valvular lesions were grouped into two categories: (a) verrucous valvular vegetations (Libman-Sachs): distinct localised masses seen on the surface of the valve leaflets; and (b) diffuse valvular thickening and stiffness, resulting in stenosis or regurgitation. ${ }^{23}$ Doppler echocardiography was performed, beginning with colour flow imaging. When abnormal intracardiac flow was detected, pulsed and continuous wave Doppler studies were performed. Mitral regurgitation was graded as mild, moderate, and severe. Regurgitation seen only with Doppler and not associated with a murmur was considered physiological. ${ }^{2425}$

\section{MEASUREMENT OF ANTIBODIES TO CARDIOLIPIN} AND $\beta_{2}$ GLYCOPROTEIN I ( $\beta_{2}$ GPI)

aCL were measured by enzyme linked immunosorbent assay (ELISA) using cardiolipin (cardiolipin 1649; Sigma; $5 \mathrm{mg} / \mathrm{ml}$ in ethanol) as antigen on polystyrene plates. ${ }^{26}$ Antibodies to $\beta_{2}$ GPI (anti- $\beta_{2}$ GPI) were measured by ELISA using $\gamma$ irradiated plates (Lindro/ Titertek; ICN Biomedical Inc, Horsham, PA, USA; Australian Nuclear Science and Techology Organisation, Sydney, Australia) incubated overnight with $\beta_{2}$ GPI $(10 \mu \mathrm{g} / \mathrm{ml}$ in phosphate buffered saline (PBS)). The non-specific binding sites were blocked with PBS/gelatin $0.5 \%$. 

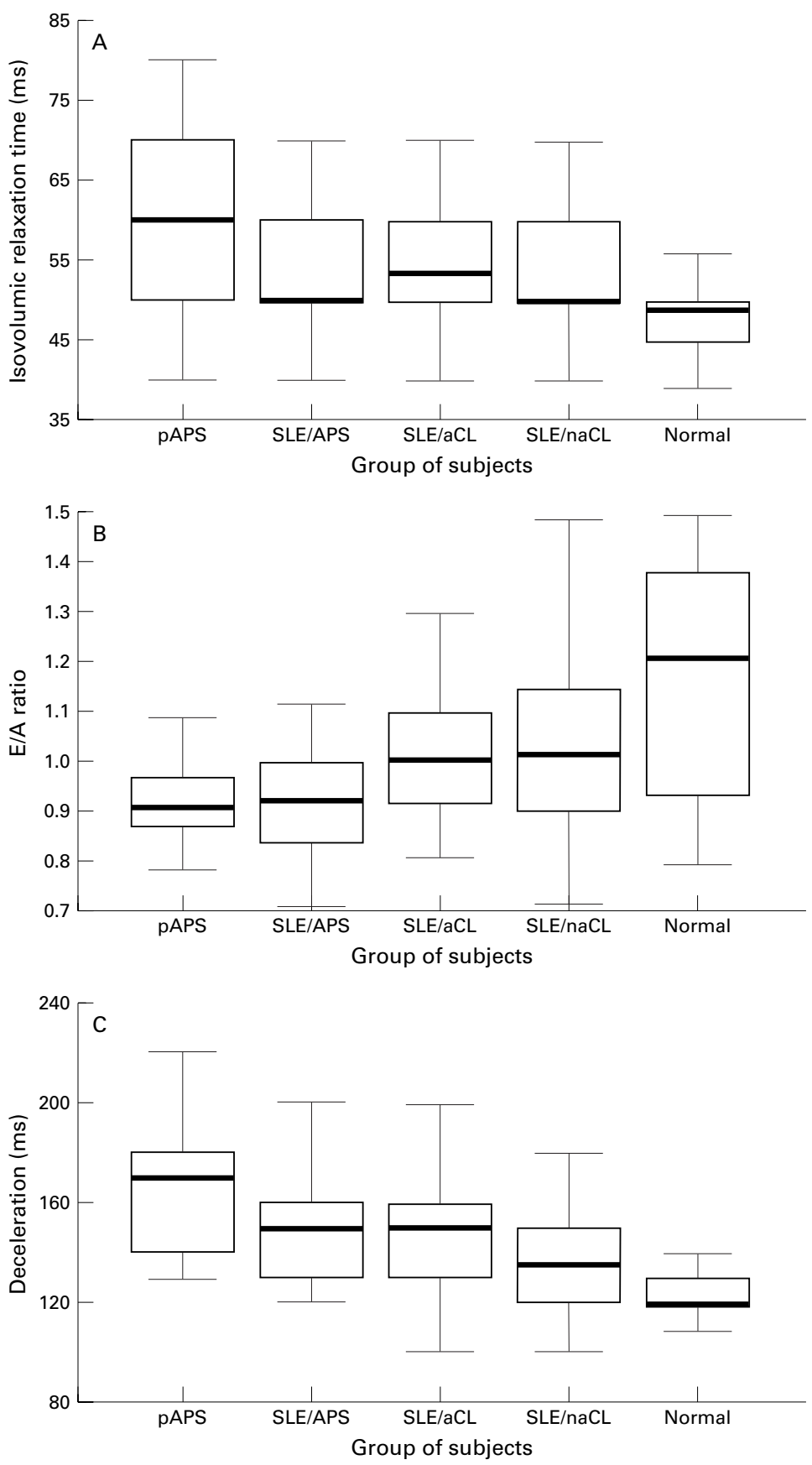

Figure 1 Box plots for right ventricular parameters, including $(A)$ isovolumic relaxation time, (B) E/A ratio, and $(C)$ deceleration time in the five groups of subjects compared. Box plots show the median (horizontal line) and the interquartile range box, together with whisker lines extending to the highest and lowest value (not showing few outliers of more than 1.5 box lengths from the box). SLE = systemic lupus erythematosus; $A P S=$ antiphospholipid syndrome; $P A P S=$ primary APS; SLE/APS = APS secondary to SLE; $S L E / a C L=S L E$ with aCL (without $A P S$ ); SLE/naCL $=S L E$ without aCL or APS.

Absorbance values more than three standard deviations above the mean of 100 normal controls were considered as positive in both assays. ${ }^{27}$

COMPARISONS AND STATISTICAL METHODS Continuous echocardiographic parameters were compared between the various groups by one way analysis of variance. Differences in the proportion of dichotomous characteristics were assessed by the $\chi^{2}$ test. For patients with aCL, regression analyses were performed considering the effects of the presence of APS, pri- mary APS, titres of IgG aCL and of IgM aCL, presence of anti- $\beta_{2}$ GPI antibodies, presence of pulmonary hypertension (pulmonary artery pressure above $30 \mathrm{~mm} \mathrm{Hg}$ ), and disease duration (from the time of probable APS diagnosis or SLE diagnosis, whichever occurred first), adjusting for age and sex, on each of the echocardiographic parameters. Linear regressions were fitted with least squares models. Analyses were conducted in SPSS. ${ }^{28}$ All p values are two tailed.

\section{Results}

CHARACTERISTICS OF STUDY PARTICIPANTS

We evaluated 143 patients and 36 healthy subjects. Among the patients, seven had a history of dyspnoea, two a history of chest pain, and eight had a history of pulmonary embolism. On examination, 21 had a systolic murmur of mitral origin, seven had a diastolic murmur, and 10 had electrocardiographic evidence of possible LV strain. A total of 108 patients had received steroids and 59 had received some other immunosuppressive treatment. Some patients were receiving angiotensin converting enzyme inhibitors $(n=22), \beta$ blockers $(n=16)$, calcium channel blockers $(n=19)$, diuretics $(n=18)$, or nitrates $(n=4)$. Key APS related manifestations included arterial thromboses other than central nervous system disease $(n=7$ patients), venous thromboses $(n=21)$, recurrent abortions $(n=13)$, autoimmune haemolytic anaemia $(n=14)$, central nervous system disease $(n=26)$, thrombocytopenia $(n=27)$, livedo reticularis $(\mathrm{n}=31)$, pulmonary embolism $(n=9)$, and leg ulcers $(n=2)$.

Fifteen patients (one with primary APS, three with secondary APS, four with SLE with aCL, and seven with SLE without aCL) were excluded from the analysis because of the presence of various exclusion criteria (15 with low FS, one of whom also had severe valvular disease; no patient had RV systolic dysfunction). Of the remaining 164 subjects, 29 had primary APS, 26 had secondary APS, 30 had aCL (but not APS) in the setting of SLE, 43 had SLE without aCL, and 36 were normal controls.

The five groups did not differ significantly in their age distribution $(p=0.7)$ and in the proportion of male patients $(p=0.15)$ (table 1$)$. The four groups of patients did not differ in the duration of disease. The majority of patients in the APS groups had raised IgG aCL, while several patients also had raised IgM; four patients only had lupus anticoagulant. Anti- $\beta_{2}$ GPI were present largely in the two APS groups.

Heart rate was not significantly different between the five groups (not shown). Five patients had mild pericardial effusions. Forty eight of the 128 patients had some evidence of valvular disease, most typically mitral regurgitation, which was detected in 44 of the 128 . Patients with aCL (with or without APS) were more likely to have some valvular disease (odds ratio (OR) 2.24, $\mathrm{p}=0.05$ ), or mitral valve regurgitation in particular (OR 3.21, $\mathrm{p}=0.005)$. The vast majority of cases had mild valve disease. Moderate mitral valve regurgitation was seen in two patients with secondary 
Table 3 Left ventricular function parameters

\begin{tabular}{|c|c|c|c|c|}
\hline \multirow[b]{2}{*}{ Patient groups* } & \multicolumn{4}{|c|}{ Echocardiographic parameters: mean (SD) } \\
\hline & $I V R T^{\star}(m s)$ & $E / A^{\star}+$ & $D T(m s)$ & $\begin{array}{l}\text { Fractional } \\
\text { shortening }\end{array}$ \\
\hline pAPS & $77.6(9.9)$ & $1.05(0.24)$ & $151.4(34.5)$ & $0.35(0.05)$ \\
\hline SLE/APS & $82.1(12.8)$ & $1.05(0.28)$ & $143.7(30.3)$ & $0.35(0.05)$ \\
\hline SLE/aCL & $77.0(8.7)$ & $1.15(0.31)$ & $146.3(35.2)$ & $0.35(0.05)$ \\
\hline SLE/no aCL & $76.6(11.3)$ & $1.04(0.26)$ & $135.0(26.0)$ & $0.35(0.05)$ \\
\hline Normal & $72.8(8.2)$ & $1.19(0.22)$ & $135.3(13.6)$ & $0.39(0.03)$ \\
\hline Comparisons & $p$ Values & & & \\
\hline All five groups & 0.015 & 0.049 & 0.079 & 0.0002 \\
\hline First four groups & 0.19 & 0.31 & 0.16 & 0.97 \\
\hline First three groups & 0.15 & 0.28 & 0.68 & 0.88 \\
\hline First two groups & 0.15 & 0.98 & 0.38 & 0.64 \\
\hline $\begin{array}{l}\text { Among patients: } \\
\text { aCL } v \text { no aCL }\end{array}$ & 0.29 & 0.35 & 0.039 & 0.97 \\
\hline Among patients with aCL: & & & & \\
\hline APS $v$ no APS & 0.26 & 0.11 & 0.85 & 0.86 \\
\hline
\end{tabular}

$\star_{\mathrm{aCL}}=$ anticardiolipin antibodies; SLE = systemic lupus erythematosus; APS = antiphospholipid syndrome; $\mathrm{pAPS}=$ primary APS; SLE/APS = APS secondary to SLE; SLE $/ \mathrm{aCL}=\mathrm{SLE}$ with aCL (without APS); SLE/no aCL = SLE without aCL or APS; IVRT = isovolumic relaxation time; E/A = ratio of $\mathrm{E}$ wave to $\mathrm{A}$ wave; $\mathrm{DT}=$ deceleration time; for definitions of groups of subjects see "Methods". †There were no formally statistically significant differences in the E wave or in the A wave alone between the five groups (not shown).

Table 4 Predictors of right ventricular (RV) diastolic function parameters among patients with anticardiolipin antibodies

\begin{tabular}{|c|c|c|}
\hline \multirow[b]{2}{*}{ Variable } & \multicolumn{2}{|l|}{ Predictors: effect ( $p$ value) } \\
\hline & Adjusted for age/sex & Multivariate \\
\hline \multirow[t]{5}{*}{ RV-E } & APS: $-4.71 \mathrm{~cm}(0.026)$ & APS: $-3.93 \mathrm{~cm}(0.052)$ \\
\hline & DD: $-0.025 \mathrm{~cm} /$ year $(0.066)$ & DD: $-0.034 \mathrm{~cm} /$ year $(0.012)$ \\
\hline & HTN: 5.04 cm (0.039) & HTN: $7.06 \mathrm{~cm}(0.0042)$ \\
\hline & Anti- $\beta_{2}$ GPI: $-3.12 \mathrm{~cm}(0.126)$ & Age: $-0.17 \mathrm{~cm} /$ year $(0.066)$ \\
\hline & Pulmonary HTN: $5.50 \mathrm{~cm}(0.105)$ & Male sex: $6.75 \mathrm{~cm}(0.014)$ \\
\hline \multirow[t]{2}{*}{ RV-A } & None & DD: $-0.041 \mathrm{~cm} /$ year $(0.032)$ \\
\hline & & HTN: $6.71 \mathrm{~cm}(0.0048)$ \\
\hline \multirow[t]{2}{*}{ RV-DT } & pAPS: $17.2 \mathrm{~ms}(0.013)$ & pAPS: $19.6 \mathrm{~ms}(0.002)$ \\
\hline & $\begin{array}{l}\text { Pulmonary HTN: } 15.6 \mathrm{~ms}(0.150) \\
\text { HTN: }-18.0 \mathrm{~ms}(0.016) \\
\text { APS: } 11.4 \mathrm{~ms}(0.093)\end{array}$ & Pulmonary HTN: $20.1 \mathrm{~ms}(0.038)$ \\
\hline RV-IVRT & IgG aCL titre: $0.89 \mathrm{~ms}(0.060)$ & IgG aCL titre: $1.10 \mathrm{~ms}(0.020)$ \\
\hline \multirow[t]{2}{*}{ RV-E/A } & APS: $-0.084(0.016)$ & APS: $-0.088(0.010)$ \\
\hline & Anti- $\beta_{2}$ GPI: $-0.073(0.029)$ & \\
\hline
\end{tabular}

aCL $=$ anticardiolipin antibodies; anti- $\beta_{2}$ GPI $=$ antibodies to $\beta_{2}$ glycoprotein I; APS $=$ antiphospholipid syndrome; DD = disease duration; HTN = hypertension; $\mathrm{pAPS}=$ primary APS . Negative values mean that the respective right ventricular variable is reduced in the presence of the predictor. In the first column, only potentially important predictors with $\mathrm{p}<0.15$, after adjusting for age and sex are shown. In the second column, predictors were selected with a backward elimination process using $p>0.10$ for variable removal and $p<0.05$ for entry. These analyses include 27 patients with primary APS, 24 patients with secondary APS, and 30 patients with SLE and aCL without APS. Four patients with APS with lupus anticoagulant, but without aCL are not included.

APS, and moderate tricuspid valve regurgitation was seen in one patient with primary APS and one patient with secondary APS. Only two patients had three valves affected (one with primary APS and one with SLE and aCL). Libman-Sacks endocarditis was seen in only four patients. Pulmonary hypertension ( $>30$ $\mathrm{mm} \mathrm{Hg}$ ) was documented in $9 / 55$ patients with APS, as compared with $5 / 73$ patients without $\operatorname{APS}(p=0.15)$.

Hypertension was more common in patients with secondary APS (12/26), while it was less common in patients with primary APS (4/29). Patients with SLE with and without aCL had a modest prevalence of hypertension ( $8 / 30$ and $7 / 43$, respectively). There was a strong association between the presence of hypertension and a history of renal disease (OR 7.0, 95\% CI 2.8 to 17.5$)$. Besides renal disease, no other causes of secondary hypertension were recorded. The cumulative duration of corticosteroid use was significantly shorter $(p=0.01)$ in patients with primary APS (mean (SD) 1 (2.4) years) than in patients with SLE (means (SD) 4.5 (5.1), 3.9
(3.9), and 3.5 (4.6) years in secondary SLE, SLE with aCL, and SLE without aCL, respectively).

ECHOCARDIOGRAPHIC PARAMETERS OF VENTRICULAR FUNCTION

In comparison with healthy controls, the groups of patients differed in all parameters of RV diastolic function (table 2). Compared with patients with SLE without APS or aCL, patients with aCL (with or without APS) had a significantly more prolonged DT and IVRT, as well as a worse $\mathrm{E}$ and $\mathrm{E} / \mathrm{A}$ ratio in the right ventricle. Within patients with SLE the presence of aCL was associated with prolonged DT and IVRT ( $p=0.03$ and $p=0.08$, respectively). Among patients with aCL, the presence of APS was associated with a worsening of most variables. Finally, within patients with APS, patients with primary APS seemed to have marginally more prolonged IVRT and DT than patients with secondary APS (table 2). Overall, the data showed a gradation of RV diastolic function impairment between normal controls, patients with SLE without aCL, patients with SLE with aCL, patients with secondary APS, and, finally, patients with primary APS (fig 1). Although patients had worse LV diastolic function variables than healthy controls, no similar gradation between patient groups could be discerned (table 3).

REGRESSION ANALYSES AMONG PATIENTS WITH ANTICARDIOLIPIN ANTIBODIES

Table 4 shows the variables that were associated with echocardiographic parameters of RV function in univariate and multivariate regression analyses. The respective analyses for $\mathrm{LV}$ parameters were not as informative (not shown). The presence of APS was associated with a worse $\mathrm{E}$ and $\mathrm{E} / \mathrm{A}$ ratio, and the presence of primary APS, in particular, was associated with a more prolonged DT, while pulmonary hypertension was the other variable that was independently associated with a prolongation of the RV-DT. Higher titres of IgG aCL were the stronger determinant of a prolonged RV-IVRT. Longer disease duration was associated with statistically significant worsening of the $\mathrm{E}$ and A parameters.

\section{Discussion}

In this study the use of Doppler echocardiography in a large cohort of patients with SLE and APS has shown an abnormal pattern of diastolic function, especially of the right ventricle, in patients with aCL. The various echocardiographic parameters reflecting diastolic dysfunction were associated with high titres of aCL, APS, disease duration, and pulmonary hypertension. RV diastolic dysfunction was found in all patient groups compared with normal controls. There was a gradation of severity, with more severe impairment in patients with primary APS, followed by the group with secondary APS and the group with SLE and aCL without APS. The gradation was particularly noticeable for DT, IVRT, and the E/A ratio-the three prime parameters reflecting 
RV diastolic function. This gradation adds further support to the hypothesis that RV diastolic dysfunction is related to aCL and the APS disease process.

High aCL titres in association with SLE or primary APS have been associated with valvular disease as well as with coronary artery thromboses or intracardiac thromboses. ${ }^{29-32} \mathrm{LV}$ systolic dysfunction may result from severe valvular disease, coronary artery disease, or diffuse cardiomyopathy. Other investigators have also described LV diastolic dysfunction despite the absence of systolic dysfunction or other cardiac disease, and the impairment of $\mathrm{LV}$ diastolic function has been associated with abnormalities in LV relaxation and myocardial disease..$^{34}$ The myocardial disease in SLE has been correlated with vasculitis, immune complex related inflammation, or, generally, the process of the disease itself and its treatment with immunosuppressive agents, including corticosteroids. ${ }^{35}$

No previous study has considered the relation of RV diastolic dysfunction with aCL or APS. In our study group, impairment of all parameters of RV diastolic function was more pronounced than the impairment of the respective LV parameters; the explanation for this may be multifactorial. If not a chance finding, this dissociation may reflect a preferential involvement or a higher susceptibility of the right ventricle. Microvascular disease may have more effect on the right ventricle, which has a substantially smaller mass than the left ventricle. Furthermore, pulmonary hypertension may also explain the predominantly RV diastolic impairment. In that context, pulmonary hypertension, together with primary APS, were the strongest independent predictors of a prolonged duration time. Pulmonary hypertension has been previously described as one of the manifestations of APS, ${ }^{36}{ }^{37}$ probably caused by recurrent pulmonary emboli or microthrombosis, and we recorded its presence in 9/55 (16\%) of the patients with APS in our study.

Thrombosis of small vessels is known to occur in APS, as it has been manifested in multiple organs (skin, brain, heart, kidney, liver, eyes).$^{38}$ Necropsies in patients with SLE with high titres of aCL, such as seen in patients with primary APS, have shown microvascular thrombosis of small intramyocardial arterioles, resulting in myocardial ischaemia and no evidence of vasculitis. ${ }^{39} 40$ aCL have been associated with small vessel thrombi and have been reported as causative factors of LV diastolic dysfunction. ${ }^{41}{ }^{42}$ Myocardial ischaemia caused by an occlusive vasculopathy of intramyocardial arteries may also explain the RV diastolic abnormality. Further studies with myocardial biopsies in patients with APS are needed to assess this hypothesis. According to our exclusion criteria, none of our patients had coronary artery disease, RV or LV systolic dysfunction, significant valvular disease, or diabetes mellitus to explain the probable diastolic impairment.

The cross sectional design of our study does not allow us to draw strong inferences about the aetiological sequence of the noticed abnor- malities. Nevertheless, despite the cross sectional study design, we noted that disease duration was significantly associated with at least two of the echocardiographic parameters of RV diastolic function, also suggesting a gradual impairment over time. A longitudinal study is underway to evaluate the evolution of diastolic dysfunction over time in the same patient group. Our study design may also have been influenced somewhat by length bias, but this is unlikely to have affected the results, except that patients in the group with aCL without APS were likely to be subjects who are less likely to progress to APS or may take longer than average to develop APS. This would have strengthened the contrast against the APS groups.

Finally, some technical caveats need to be considered. LV and particularly RV inflow velocities may be influenced by multiple factors, including heart rate, age, loading conditions, ventricular interaction, and diastolic "suction". ${ }^{43}$ However, in our group there were no significant differences in heart rate, age, and LV mass between the five groups. Additionally, the results were similar when the analysis was limited to patients without systemic hypertension. A source of error is the beat-to-beat variability in flow dynamics, especially on the right side, due to respiration. To minimise errors and improve reproducibility of measurements we averaged multiple consecutive beats. ${ }^{43}$ Another study limitation is the lack of assessment of hepatic and pulmonary venous flow.

Doppler echocardiography is a simple, noninvasive technique, widely used to detect subtle, asymptomatic myocardial abnormalities. Our study suggests that it may be used in patients with aCL or APS for the early detection of diastolic dysfunction, in particular of the right ventricle. The clinical significance of this dysfunction needs to be established in long term studies. Further research is also needed to determine whether the detection of such abnormalities in otherwise asymptomatic subjects should affect the therapeutic management of patients with aCL.

1 Hughes GRV. The anticardiolipin syndrome. Clin Exp Rheumatol 1985;3:285-6.

2 Alarcon-Segovia D, Sanchez-Guerrero J. Primary antiphospholipid syndrome. J Rheumatol 1989;16:482-8.

3 Libman E, Sachs B. A hitherto undescribed form of valvular and mitral endocarditis. Arch Intern Med 1924;33:70137.

4 Paget SA, Bulkley BH, Grauer LE, Seningen R. Mitral valve disease of systemic lupus erythematosus. A cause of severe congestive heart failure reversed by valve replacement. Am J Med 1975;59:134-9.

5 Klinkhoff AV, Thompson CR, Reid GD, Tomlinson CW. $M$-mode and two-dimensional echocardiographic abnormalities in systemic lupus erythematosus. JAMA 1985;253: 3273-7.

6 Chartash EK, Lans DM, Paget SA, Qamar T, Lockshin MD. Aortic insufficiency and mitral regurgitation in patients with systemic lupus erythematosus and the antiphospholipid syndrome. Am J Med 1989;86: $407-12$

7 Hamstein A, Norberg R, Bjorkholm M, De Faire U, Holm $\mathrm{G}$. Antibodies to cardiolipin in young survivors of myocardial infarction. Lancet 1986;i:113-15.

8 Lubbe WF, Asherson RA. Intracardiac thrombus in systemic lupus erythematosus associated with lupus systemic lupus erythematosus associated with

9 Leventhal LJ, Borofsky MA, Bergey PD, Schumacher HR. Antiphospholipid antibody syndrome with right atrial Antiphospholipid antibody syndrome with right atrial
thrombosis mimicking an atrial myxoma. Am J Med 1989; 87:111-13. 
10 Hasnie AM, Stoddard MF, Gleason CB, Wagner SG, Longaker RA, Pierangeli S, et al. Diastolic dysfunction is a feature of the antip

11 Nihoyannopoulos P, Gomez PM, Joshi J, Loizou S, Walport MJ, Oakley CM. Cardiac abnormalities in systemic lupus erythematosus. Association with raised anticardiolipin antibodies. Circulation 1990;82:369-75.

12 Alarcon-Segovia D, Perez-Vazquez ME, Villa AR, Drenkard C, Cabiedes J. Preliminary classification criteria for the anticardiolipin syndrome within systemic lupus erythematosus. Semin Arthritis Rheum 1992;21:275-86.

13 Tan EM, Cohen AS, Fries JF, Masi AT, McShane DJ, Roth field NF, et al. The 1982 revised criteria for the classification of lupus erythematosus. Arthritis Rheum 1982;25:1271-7.

14 Tajik AJ, Seward JB, Hagler DJ, Mair DD, Lie JT Two-dimensional real-time ultrasonic imaging of the heart and great vessels: technique, image orientation, structure and great vessels: technique, image orientation, structure 271-303.

15 Devereux RB, Alonso DR, Lutas EM, Gottlieb GJ, Campo $\mathrm{E}$, Sachs I, et al. Echocardiographic assessment of left ventricular hypertrophy. Comparison to necropsy findings. Am J Cardiol 1986;57:450-8.

16 Pai RG, Bansal RC, Shah PM. Determinants of the rate of right ventricular pressure rise by Doppler echocardiography: potential value in the assessment of right ventricular function. J Heart Valve Dis 1994;3:17984.

17 Kaul S, Tei C, Hopkins JM, Shah PM. Assessment of right ventricular function using two dimensional echocardiography. Am Heart J 1984;107:526-31.

18 Karatasakis GT, Karagounis LA, Kolyvas PA, Manginas A, Athanassopoulos GD, Aggelakas SA, et al. Prognostic significance of echocardiographically estimated right ventricular shortening in advanced heart failure. Am J Cardiol 1998;82:329-34.

19 Nishimura RA, Miller FA, Callahan MJ, Benassi RC, Seward JB, Tajik AJ. Doppler echocardiography: theory,
instrumentation, technique and application. Mayo Clinic instrumentation, techniqu

20 Spirito P, Maron BJ, Bonow RO. Noninvasive assessment of left ventricular diastolic function: comparative analysis of Doppler echocardiography and radionuclide angiographic techniques. J Am Coll Cardiol 1986;7:518-26.

21 Rokey R, Kuo LC, Zoghbi WA, Limacher MC, Quinones MA. Determination of parameters of left ventricular diastolic filling with pulsed Doppler echocardiography: comparison with cineangiography. Circulation 1985;71: 543-50.

22 Reynolds T. The echocardiographer pocket reference. Phoenix, USA: Arizona Heart Institute Foundation, 1993:152-3.

23 Galve E, Riera J, Pigrau C, Miralda G, Castillo H, Soler SY. Prevalence, morphologic types and evolution of cardiac valvular disease in systemic lupus erythematosus. N Engl J Med 1988;319:817-23.

24 Switzer DF, Nanda NC. Color Doppler evaluation of valvular regurgitation. Echocardiography 1985;2:533.

25 Yoshida K, Yoshikawa J, Shakudo M, Akasaka T, Jyo Y, Takao S, et al. Color Doppler evaluation of valvular regurTakao S, et al. Color Doppler evaluation of valvular regur-

26 Manoussakis MN, Gharavi AE, Drosos AA, Kitridou RC Moutsopoulos HM. Anticardiolipin antibodies in unselected autoimmune rheumatic disease patients. Clin
27 Vlachoyiannopoulos PG, Petrovas C, Tektonidou M, Krilis S, Moutsopoulos HM. Antibodies to $\beta 2$-glycoprotein-I: urea resistance, binding specificity, and associan
thrombosis. J Clin Immunol 1998;18:380-91.

28 Advanced SPSS, version 6.1. Chicago: SPSS Inc, 1994.

29 Brenner B, Blumenfeld Z, Markiewicz W, Reisner SA. Cardiac involvement in patients with primary antiphospholipid syndrome. J Am Coll Cardiol 1991;18:931-6.

30 Gleason CB, Stoddard MF, Wagner SG, Longaker RA, Pierangeli S, Harris EN. A comparison of cardiac involvement in the primary antiphospholipid syndrome versus anticardiolipin-negative systemic lupus erythematosus. Am Heart J 1993;125:1123-9.

31 Galve E, Ordi J, Barquiero J, Evangelista A, Vilardell M, Soler-Soler J. Valvular heart disease in the primary antiphospholipid syndrome. Ann Intern Med 1992;116: 293-8.

32 Khamashta MA, Cervera R, Asherson RA, Font J, Gil A, Coltart DJ, et al. Association of antibodies against phospholipids with heart valve disease in systemic lupus erythematosus. Lancet 1990;335:1541-4.

33 Coudray N, de Zuttere D, Bletry O, Piette JC, Wechsler B, Godeau $\mathrm{P}$, et al. $\mathrm{M}$ mode and Doppler echocardiographic assessment of left ventricular diastolic function in primary antiphospholipid syndrome. Br Heart J 1995;74:531-5.

34 Leung WH, Wong KL, Lau CP, Wong CK, Cheng CH, Tai YI. Doppler echocardiographic evaluation of left ventricular diastolic function in patients with systemic lupus erythematosus. Am Heart J 1990;120:82-7.

35 Bidani AK, Roberts JL, Schwartz MM, Lewis EJ. Immunopathology of cardiac lesions in fatal systemic lupus erythematosus. Am J Med 1980;69:849-58.

36 Asherson RA, Oakley CM. Pulmonary hypertension and systemic lupus erythematosus. J Rheumatol 1986;13:1-5.

37 Brucato A, Bandu F, Barberis M, Redaelli R, Casadei G, Allegri $\mathrm{F}$, et al. Pulmonary hypertension secondary to thrombosis of the pulmonary vessels in a patient with the primary antiphospholipid syndrome. J Rheumatol 1994; 21:942-4.

38 Hughes GRV. The antiphospholipid syndrome: ten years on. Lancet 1993;342:341-4.

39 Greisman SG, Thayaparan RS, Godwin TA, Lockshin MD. Occlusive vasculopathy in systemic lupus erythematosus. Arch Intern Med 1991;15:389-92.

40 Brown JH, Doherty CC, Allen DC, Morton P. Fatal cardiac failure due to myocardial microthrombi in systemic lupus erythematosus. BM J 1988;296:1505.

41 Murphy JJ, Leach IH. Findings at necropsy in the heart of a patient with anticardiolipin syndrome. Br Heart J 1989;62: 61-4.

42 Leung WH, Wong KL, Lau CP, Wong CK, Liu HW. Association between antiphospholipid anntibodies and cardiac abnormalities in patients with systemic lupus erythematosus. Am J Med 1990;89:411-19.

43 Zoghbi WA, Habib GB, Quinones MA. Doppler assessment of right ventricular filling in a normal population. Comparison with left ventricular filling dynamics. Circulation 1990;82:1316-24.

44 Pye MP, Pringle SD, Cobbe SM. Reference values and reproducibility of Doppler echocardiography in the assessment of the tricuspid valve and right ventricular diastolic function in normal subjects. Am J Cardiol 1991;67:26973 